\title{
Drive System Parameters Influence on Run-Up Process of the Drilling Rig Pumping Unit
}

\author{
Roman Kovalchuk'1, Yuriy Molkov², Taras Lenkovskiy², Oleksandr Grytsenko³, \\ Volodymyr Krasinskyi ${ }^{3}$, Tomasz Garbacz ${ }^{4^{*}}$
}

1 Hetman Petro Sahaidachnyi National Army Academy, Department of Engineering Mechanics, 32 Heroes of Maidan St., Lviv 79012, Ukraine

2 Karpenko Physico-Mechanical Institute of the NAS of Ukraine, Department of Strength and Durability of Structures under Complex Loading, 5 Naukova St., Lviv 79060, Ukraine

3 Lviv Polytechnic National University, Department of Chemical Technology and Plastics Processing, Bandera 12 St., 79013 Lviv, Ukraine

${ }^{4}$ Lublin University of Technology, Faculty of Mechanical Engineering, Department of Polymer Processing, ul. Nadbystrzycka 36, 20-618 Lublin, Poland

* Corresponding author's e-mail: t.garbacz@pollub.pl

\begin{abstract}
A mathematical model of dynamic processes in pumping unit of a drilling rig with friction clutch, taking into account variable moment of inertia of slider-crank mechanism links and random number of pistons of the pump, was developed. Joint integration of differential equations of motion of pumping unit parts was carried out with numerical methods. The calculations performed considered the torque moment of friction in the clutch and equations of electromagnetic processes in asynchronous motor. The results of research of run-up processes of pumping unit were presented. The influence of parameters of friction clutch on the performance of pumping unit was established.
\end{abstract}

Keywords: dynamics of machines, non-stable modes of operation, run-up process, friction clutch, pumping unit, dynamic load

\section{INTRODUCTION}

Pumping units of drilling rigs work under high dynamic loadings. Rational design and exploiting parameters of these machines can be defined only on the basis of a comprehensive study of oscillation processes as well as an investigation of a stress-strain state of their parts. The complexity of pumping units as technical systems, a repeating character of functioning, and clearly expressed interdependence of phenomena of different physical nature require developing special methods of dynamic calculations. In view of the necessity to simultaneously ensure high productivity of circulatory systems of drilling rigs, as well as durability and reliability of structure elements, particular significance was rendered to improve the analysis methodology of non-steady operational modes of pumping units.

Productivity and reliability of a drilling rig considerably depend on an efficient operation of a pumping unit which provides moving debris to the surface by means of drilling fluid injection into a well. The investigation of dynamics of pumping units is directly connected with the definition of their summary inertia moments as functions of rotational angles of root shafts and it was reviewed by Yutaev [11]. The basics of dynamics of mechanisms and machines were presented by Dresig et al. [3, 9, 1]. In the papers written by Erkaya et al. [4] and Mudrik [8], the theoretical bases of mathematic modeling of mechanical systems dynamics with variable inertia characteristics of links and investigation examples of 
such systems were presented. Theoretical and practical issues of kinematics and dynamics of crank-and-slider mechanisms as well as reducing of masses of mechanisms and structure elements were considered in the investigations by Fung et al. [5, 10, 12, 7]. According to Kharchenko's [6] approach, a calculation process based on compatible integration of differential equations of movement, written taking into account the instability of a summary inertia moment of crank-and-slider pumping mechanisms, and equations of electromagnetic processes in an asynchronous motor was proposed. The vibration phenomena of drill string were investigated by Christoforou [2].

Nevertheless, the influence of friction clutch on the dynamic loading of the elements of pumping unit has not been investigated enough. Hence, there is a need for carrying out a detailed analysis of the dynamic phenomena in pumping unit and its drive with the purpose of rational selection of operational parameters of the friction clutches, which would provide the improvement of circulation system components strength and reliability. In this study, a mathematical model of dynamic processes in a pumping unit with variable number of pistons and the friction clutch, considering inconstancy of inertia moments of the slidercrank mechanism of the pump and connection of mechanical and electromagnetic oscillatory processes was developed. The joint integration of the differential equations of movement of the mechanical system of variable structure as well as the equations of the electromagnetic phenomena in the asynchronous motor gives an opportunity to analyze the processes taking place at the run-up and at the stable mode of drilling pumps operation.

\section{DIFFERENTIAL EQUATIONS OF THE PUMPING UNIT MOVEMENT}

The mechanical system of the pumping unit, which consists of an asynchronous motor, an air clutch, a belt gear, a reducer and piston pump is illustrated schematically in Fig. 1, where: $J_{1}-$ a reduced moment of inertia of motor's rotor with the driving part of the air clutch; $J_{2}$ - a reduced moment of inertia of the air clutch driven part with a shaft and driving pulley of the belt gear; $J_{3}-$ a reduced moment of inertia of transmission shaft with a gear-wheel and a driven pulley of the belt gear; $J_{4}$ - a reduced moment of inertia of the slider-crank mechanism of the pump; $c_{1}$ - reduced rigidity of the cone belts; $v_{1}$ - reduced coefficient of the belts damping; $c_{2}$ - reduced rigidity of the gear reduction unit; $v_{2}$ - reduced coefficient of the gear drive damping; $M_{E}$ - reduced electromagnetic moment of a motor; $M_{d}-$ movement drag moment, which has an effect on the driving shaft of a pump; $\varphi_{1}, \varphi_{2}, \varphi_{3}, \varphi_{4}$ - reduced angular coordinates. Inertial and elastic-dissipative parameters of the mechanical system are reduced to driving shaft of a pump.

Equations of the unit elements movement are composed similarly to the Lagrange's equations of the second kind

$$
\frac{d}{d t}\left(\frac{\partial T}{\partial \dot{q}_{j}}\right)-\frac{\partial T}{\partial q_{j}}+\frac{\partial \Pi}{\partial q_{j}}+\frac{\partial \Phi}{\partial \dot{q}_{j}}=Q_{j} \quad(j=1,4),
$$

where: $T$ and $\Pi$ are the kinetic and potential energies of the mechanical system; $\Phi$ - the Rayleigh dissipation function; $Q_{j}$ - generalized force; $q_{j}-$ generalized coordinate; $t$ - time.

Designating as the generalized coordinates the following values

$$
q_{1}=\varphi_{1} ; \quad q_{2}=\varphi_{2} ; q_{3}=\varphi_{3} ; q_{4}=\varphi_{4},
$$

the kinetic energy of the system is presented as

$$
T=\frac{J_{1} \omega_{1}{ }^{2}}{2}+\frac{J_{2} \omega_{2}{ }^{2}}{2}+\frac{J_{3} \omega_{3}{ }^{2}}{2}+\frac{J_{4} \omega_{4}{ }^{2}}{2},
$$

where: $\omega_{1}, \omega_{2}, \omega_{3}, \omega_{4}-$ an angular velocity of the electric motor's rotor, an angular velocity of the half-clutch, joined with the driving

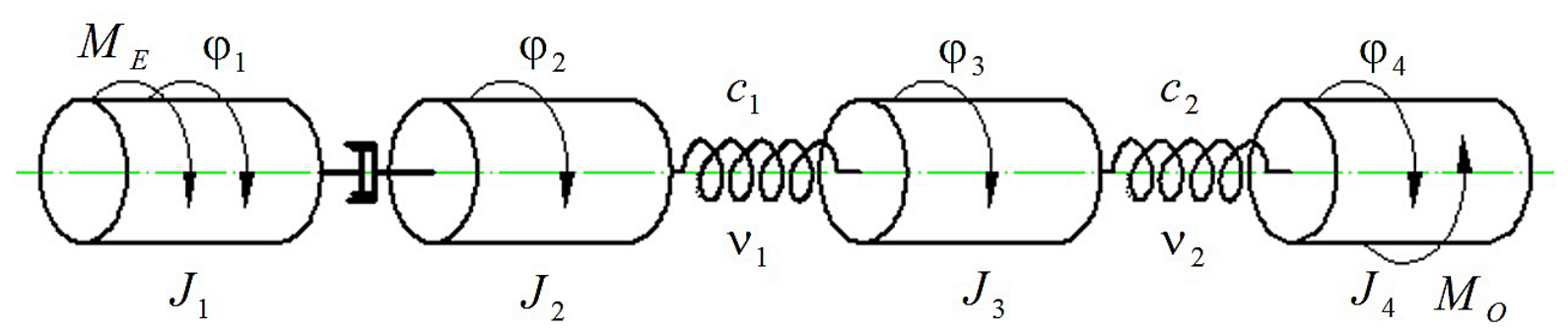

Fig. 1. Scheme of the mechanical system of pumping unit 
pulley of the belt gear, an angular velocity of the high-speed shaft of the reducer, joined with driven pulley of the belt gear and a real angular velocity of the root shaft of the pump, respectively, reduced to the root shaft,

$$
\frac{d \varphi_{1}}{d t}=\omega_{1}, \quad \frac{d \varphi_{2}}{d t}=\omega_{2}, \frac{d \varphi_{3}}{d t}=\omega_{3}, \frac{d \varphi_{4}}{d t}=\omega_{4} .
$$

The potential energy and the Rayleigh dissipation function with consideration of the dependencies (2), (4) are written as

$$
\begin{gathered}
\Pi=\frac{c_{1}\left(\varphi_{2}-\varphi_{3}\right)^{2}}{2}+\frac{c_{2}\left(\varphi_{3}-\varphi_{4}\right)^{2}}{2} ; \\
\Phi=\frac{v_{1}\left(\omega_{2}-\omega_{3}\right)^{2}}{2}+\frac{v_{2}\left(\omega_{3}-\omega_{4}\right)^{2}}{2} .
\end{gathered}
$$

For the mode with sliding in the air clutch, taking derivations of formulae (3) and (5) and inserting them in the Lagrange's equations of the second kind (1), we receive the equations of motion written as follows:

$$
\begin{gathered}
J_{1} \frac{d \omega_{1}}{d t}=M_{E 3}-M_{f} ; \\
J_{2} \frac{d \omega_{2}}{d t}=M_{f}-c_{1}\left(\varphi_{2}-\varphi_{3}\right)-v_{1}\left(\omega_{2}-\omega_{3}\right) ; \\
J_{3} \frac{d \omega_{3}}{d t}=c_{1}\left(\varphi_{2}-\varphi_{3}\right)+v_{1}\left(\omega_{2}-\omega_{3}\right)-c_{2}\left(\varphi_{3}-\varphi_{4}\right)-v_{2}\left(\omega_{3}-\omega_{4}\right) ; \\
J_{4} \frac{d \omega_{4}}{d t}=-\frac{1}{2} \frac{\partial J_{4}}{\partial \varphi_{4}} \omega_{4}^{2}+c_{2}\left(\varphi_{3}-\varphi_{4}\right)+v_{2}\left(\omega_{3}-\omega_{4}\right)-M_{d},
\end{gathered}
$$

where: $M_{f}-$ a moment of friction in the air clutch, reduced to the root shaft, which is changed according to the law:

$$
\begin{aligned}
& M_{f}=\alpha_{t} \cdot t, \text { if } t<t_{r} ; \\
& M_{f}=\alpha_{t} \cdot t_{r}, \text { if } t \geq t_{r},
\end{aligned}
$$

where: $t_{r}$-clutch filling time;

$\alpha_{t}$-coefficient, which is determined by the dependence

$$
\alpha_{t}=\frac{M_{f 0}}{t_{r}}
$$

where: $M_{f 0}-$ maximum moment of friction in a clutch.

The reduced moment of the motor is found according to the formula

$$
M_{E}=M_{E 0} \cdot u,
$$

where: $M_{E 0}$-real electromagnetic moment of the motor; $u$-gear-ratio of the drive.
If the sliding in the air clutch is absent, the links with the moments of inertia $J_{1}$ and $J_{2}$ perform a cooperative motion. The equation of the system motion is obtained by substitution of the formulae (3) and (5) to the relation (1) assuming that $\varphi_{1}=\varphi_{2}$,

$$
\begin{gathered}
\omega_{1}=\omega_{2} ; \varphi_{1}=\varphi_{1}\left(t_{1}\right)+\varphi_{2}(t)-\varphi_{2}\left(t_{1}\right) \\
\left(J_{1}+J_{2}\right) \frac{d \omega_{2}}{d t}=M_{E}-c_{1}\left(\varphi_{2}-\varphi_{3}\right)-v_{1}\left(\omega_{2}-\omega_{3}\right) ; \\
J_{3} \frac{d \omega_{3}}{d t}=c_{1}\left(\varphi_{2}-\varphi_{3}\right)+v_{1}\left(\omega_{2}-\omega_{3}\right)-c_{2}\left(\varphi_{3}-\varphi_{4}\right)-v_{2}\left(\omega_{3}-\omega_{4}\right) \\
J_{4} \frac{d \omega_{4}}{d t}=-\frac{1}{2} \frac{\partial J_{4}}{\partial \varphi_{4}} \omega_{4}^{2} \\
+c_{2}\left(\varphi_{3}-\varphi_{4}\right)+v_{2}\left(\omega_{3}-\omega_{4}\right)-M_{d}
\end{gathered}
$$

The transition from the motion mode with sliding to the mode without sliding is fulfilled to meet the following conditions

$$
\begin{gathered}
\omega_{2}=\omega_{1}, \\
M_{E}-J_{1} \frac{d \omega_{1}}{d t} \leq M_{f} .
\end{gathered}
$$

If the condition expressed by second relation (9) is violated at some moment of the system movement without sliding in the clutch, then the sliding arises again.

In the course of numerical integration of the differential equations (4), (6) and (4), (8), it is necessary to define the derived function $J_{4}$ by the coordinate $\varphi_{4}$ and electromagnetic moment of the motor $M_{E}$ at every step.

The reduced moment of inertia of the mechanism of the pump, which includes $n$ of the slidercrank mechanisms, is written as:

$$
\begin{aligned}
& J_{R}(\varphi)=\sum_{i=1}^{n}\left[J_{S 1}+m_{1} a_{1}^{2}+m_{2} u_{i} \frac{l_{1}^{2}\left(\cos \varphi_{i}\right)^{2}}{h_{i}}+J_{S 2} \frac{l_{1}^{2}\left(\cos \varphi_{i}\right)^{2}}{h_{i}}+\right. \\
& \left.+m_{3}\left(-l_{1}\left(\sin \varphi_{i}+\frac{l_{1} \sin 2 \varphi_{i}}{2 \sqrt{h_{i}}}\right)\right)\right]
\end{aligned}
$$

where:

$$
h_{i}=l_{2}^{2}-l_{1}^{2}\left(\sin \varphi_{i}\right)^{2}, u_{i}=
$$

$=\frac{h_{i}}{\left(\cos \varphi_{i}\right)^{2}}+a_{2}{ }^{2}-2 a_{2} \frac{h_{i}}{l_{2}}+2 a_{2} \frac{l_{1} \cdot\left(\sin \varphi_{i}\right)^{2} \cdot \sqrt{h_{i}}}{\cos \varphi_{i} \cdot l_{2}} ;$

provided that $\varphi_{\mathrm{i}}(i=1,2, \ldots, n)$ - the angular coordinates of a crank of single slider-crank mechanism; $m_{1}, m_{2}, m_{3}$ - masses of the crank, connecting-rod and a piston; $J_{\mathrm{S} 1}$ and $J_{\mathrm{S} 2}-$ central moment of inertia of the crank shaft and a connecting-rod; $l_{1}$ and $l_{2}$ - linear dimensions of the 
links; $a_{1}-$ a distance from the gravity center of the crank shaft to the axle of its rotation; $a_{2}-$ a distance from the gravity center of the connectingrod to the axle of its connection to the crank shaft.

The rotation angles of the driving links of the slider-crank mechanisms of the pump $\varphi_{i}(i=1,2, \ldots, n)$ are related to the rotation angle of the driving shaft of a pump $\varphi$ by the following dependencies:

- for a single-cylinder pump

- $\varphi_{1}=\varphi$

- for a double-cylinder single-acting pump

$$
\varphi_{1}=\varphi, \quad \varphi_{2}=\varphi+\pi
$$

- for a double-cylinder double-acting pump

$$
\varphi_{1}=\varphi, \quad \varphi_{2}=\varphi+\frac{\pi}{2}
$$

- for a triple-cylinder single-acting pump

$$
\varphi_{1}=\varphi, \quad \varphi_{2}=\varphi+\frac{2}{3} \pi, \quad \varphi_{3}=\varphi+\frac{4}{3} \pi \text {. }
$$

The derivative $d J_{R} / d \varphi$ is a sum of derivatives $d_{i}$ from formula (11), which depends on the number $n$ of the slider-crank mechanisms of the pump and displacement of rotation angles of the driving links of these mechanisms.

$$
\frac{d J_{R}}{d \varphi}=\sum_{i=1}^{n} d_{i}
$$

A force moment of the effective resistance of the driving shaft is determined according to the formula

$$
M_{d}=\sum_{i=1}^{n} M_{d i},
$$

where: $M_{d i}-$ a moment of effective resistance, which creates a fluid pressure on every $i$ piston,

$$
M_{d i}=P_{i} l_{1} \Theta_{i},
$$

provided that $P_{i}$ is a pressure force on the piston, and $\Theta_{i}$ - trigonometric functions of the rotation angle of the crank shaft.

For double-action pump, $P_{i}$ is determined according to the dependence:

$$
\begin{aligned}
& P_{i}=-p F_{p}, \text { if } v_{i}>0 ; P_{i}=0, \text { if } v_{i}=0 ; \\
& P_{i}=-p F_{p}, \text { if } v_{i}>0 ; P_{i}=0, \text { if } v_{i}=0 ; \\
& P_{i}=p\left(F_{p}-F_{r}\right), \text { if } v_{i}<0,
\end{aligned}
$$

where: $F_{p}$ and $F_{r}$ are the areas of a cross-section of a piston and a rod,

$p$ is a fluid pressure on the piston; $V_{i}-$ piston velocity.

$$
v_{i}=-\omega_{2} l_{1} \Theta_{i} .
$$

Trigonometric function of the turning angle of the crankshaft $\Theta_{i}$ from dependencies (15), (17), is written as:

$$
\Theta_{i}=\sin \varphi_{i}+\frac{\sin \varphi_{i} \cos \varphi_{i}}{\sqrt{\left(\frac{l_{2}}{l_{1}}\right)^{2}-\left(\sin \varphi_{i}\right)^{2}}} .
$$

A real electromagnetic moment of a motor $M_{E 0}$ is determined based on the equations of the electromagnetic processes in the asynchronous motor.

The equations of the electromagnetic processes in the asynchronous motor

The electromagnetic phenomena in the asynchronous motor with consideration of magnetic circuit saturation are described by the following equations [1]:

$$
\begin{aligned}
& \frac{d i_{S}}{d t}=A_{S}\left(u+\Omega_{S} \Psi_{S}-R_{S} i_{S}\right)+B_{S}\left(\Omega_{R} \Psi_{R}-R_{R} i_{R}\right) ; \\
& \frac{d i_{R}}{d t}=A_{R}\left(\Omega_{R} \Psi_{R}-R_{R} i_{R}\right)+B_{R}\left(u_{S}+\Omega_{S} \Psi_{S}-R_{S} i_{S}\right),
\end{aligned}
$$

where: $i_{S^{\prime}} i_{R}$ and $u_{S}$ - matrix columns of the currents and voltages;

$A_{S^{\prime}} B_{S^{\prime}} A_{R^{\prime}} B_{R}$ - the connection matrices; $\Omega_{S^{\prime}}, \Omega_{R}$ - rotation frequency matrices; $\Psi_{S^{\prime}} \Psi_{R}-$ magnetic linkage matrix columns.

Index $S$ indicates belonging of the value to the stator winding, and $R$ - to the rotor winding. Matrix-columns $i_{S}, i_{R}, u_{S}$ are determined with the following equations

$$
i_{j}(j=S, R)=\operatorname{col}\left(i_{\dot{x}^{\prime}}, i_{\dot{j}^{\prime}}\right) ; \quad u_{S}=\operatorname{col}\left(U_{m}, 0\right),
$$
where: $i_{j x}, i_{j y}-$ a projection of currents on coordinate axes $x, y$;

$U_{m}$ - voltage amplitude of the power circuit.

Square matrices $A_{S}, B_{S}, A_{R}, B_{R}$ are determined by means of the following dependences

$$
\begin{gathered}
A_{S}=\alpha_{S}\left(1-\alpha_{S} G\right) ; \quad B_{S}=-\alpha_{S} \alpha_{R} G ; \\
A_{R}=\alpha_{R}\left(1-\alpha_{R} G\right) ; \quad B_{R}=B_{S},
\end{gathered}
$$$$
\text { where: }
$$ 


$$
G=\frac{1}{i_{m}{ }^{2}}\left[\begin{array}{ll}
R i_{x}{ }^{2}+T i_{y}{ }^{2} & (R-T) i_{i} i_{y} \\
(R-T) i_{x} i_{y} & T i_{x}{ }^{2}+R i_{y}{ }^{2}
\end{array}\right],
$$

provided that

$$
R=\frac{1}{\rho+\alpha_{S}+\alpha_{R}} ; \quad T=\frac{1}{\tau+\alpha_{S}+\alpha_{R}} .
$$

where: $i_{m^{\prime}}, i_{x^{\prime}} i_{y}-$ the magnetized current and its components along the axes $x, y ; \tau$,

$\rho$ - the values determined from the magnetizing curve that is a functional dependence of the operating magnetic flux linkage $\Psi_{m}$ on the magnetizing current;

$\alpha_{S^{\prime}} \alpha_{R}{ }^{m}-$ the values, reciprocal to dissipation inductances of stator and rotor winding.

Rotation frequency matrices

$$
\Omega_{S}=\left[\begin{array}{cc}
0 & \omega_{0} \\
-\omega_{0} & 0
\end{array}\right] ; \quad \Omega_{R}=\left[\begin{array}{cc}
0 & \omega_{0}-\omega_{R} \\
\omega_{R}-\omega_{0} & 0
\end{array}\right],
$$

where: $\omega_{0}$ and $\omega_{R}-$ a synchronous angular velocity of motor and the angular velocity of the rotor are expressed in electrical radians per second.

The values $\omega_{0}$ and $\omega_{R}$ are following

$$
\omega_{0}=314 ; \omega_{R}=\omega_{1} \cdot u \cdot p_{0},
$$

for the second stage

$$
\omega_{R}=\omega_{2} \cdot u \cdot p_{0},
$$

where: $u-$ a gear ratio;

$p_{0}$ - number of magnetic pole pairs.

The column matrices of complete magnetic flux linkages of the stator and rotor winding are written as:

$$
\Psi_{S}=\frac{1}{\alpha_{S}} i_{S}+\frac{1}{\tau} i ; \quad \Psi_{R}=\frac{1}{\alpha_{R}} i_{R}+\frac{1}{\tau} i,
$$

where:

$$
\begin{aligned}
& i=\operatorname{col}\left(i_{x}, i_{y}\right) . \\
& \text { Values } \\
& i_{x}=i_{S x}+i_{R x} ; i_{y}=i_{S y}+i_{R y} ; i_{m}=\sqrt{i_{x}^{2}+i_{y}{ }^{2}}
\end{aligned}
$$

Values $\tau$ and $\rho$ are expressed by the following formula

$$
\tau=\frac{i_{m}}{\psi_{m}} ; \quad \rho=\frac{d i_{m}}{d \psi_{m}}
$$

The electromagnetic moment is found according to the following formula

$$
M_{E}=M_{E 0} \cdot u=\frac{3}{2} \cdot u \cdot p_{0} \frac{1}{\tau}\left(i_{R x} i_{S y}-i_{R y} i_{S x}\right) .
$$

The magnetizing curve is written as:

$$
\begin{gathered}
\psi_{m}=a_{1} i_{m}+a_{2} i_{m}{ }^{3}+a_{3} i_{m}{ }^{5}, \text { if } i_{m}>i_{m k} ; \\
\psi_{m}=\alpha_{m}{ }^{-1} i_{m}, \text { if } i_{m} \leq i_{m k},
\end{gathered}
$$

where: $i_{m k}-$ a critical value of the magnetizing current, beyond the range of which a $\psi_{m}\left(i_{m}\right)$ dependence is nonlinear.

Then $\tau$ and $\rho$, according to the formulae (20), are determined by the dependencies

$$
\begin{aligned}
& \tau=\left(a_{1} i_{m}+a_{2} i_{m}{ }^{2}+a_{3} i_{m}{ }^{4}\right)^{-1}, \text { if } i_{m}>i_{m k} ; \\
& \tau=\alpha_{m}, \text { if } i_{m} \leq i_{m k}
\end{aligned}
$$

$$
\begin{aligned}
& \rho=\left(a_{1} i_{m}+3 a_{2} i_{m}{ }^{2}+5 a_{3} i_{m}{ }^{4}\right)^{-1}, \text { if } i_{m}>i_{m k} ; \\
& \rho=\alpha_{m}, \text { if } i_{m} \leq i_{m k} .
\end{aligned}
$$

For numerical integration of the differential equations (19) there is no need for the information on the magnetizing curve (22), as long as the formulae (23) and (24) are used in the course of calculation. In case of sliding $-\omega_{R}=\omega_{1} \cdot u$; in case of sliding absence $\omega_{R}=\omega_{2} \cdot u$.

\section{INITIAL CONDITIONS OF DIFFERENTIAL EQUATIONS INTEGRATING}

Projections of currents on a coordinate axes at the beginning of idle engine start equals zero but in this particular case, we accept the initial conditions that the motor has been operating in a stable mode without any loading, in other words

$$
i_{S x}(0)=i_{S x 0} ; i_{S y}(0)=i_{S y 0} ; i_{R x}(0)=i_{R x 0} ; i_{R y}(0)=i_{R y 0},
$$

where: $i_{S x 0^{\prime}} i_{S y 0^{\prime}} i_{R x 0^{\prime}} i_{R y 0}$ - current values for the particular instants of time of the idle mode of the motor.

We receive the values of currents projection by solving a system of equations (19) and motion equations of motor's rotor.

Initial conditions of integrating of equations (4), (6) and (4), (8) are written as:

$$
\begin{aligned}
& \varphi_{1}(0)=0 ; \varphi_{2}(0)=0 ; \varphi_{3}(0)=0 ; \varphi_{4}(0)=0 ; \\
& \omega_{1}(0)=\omega_{10} ; \omega_{2}(0)=0 ; \omega_{3}(0)=0 ; \omega_{4}(0)=0 .
\end{aligned}
$$


The value of angular velocity $\omega_{10}$ at the instant $t=0$ corresponds to the idle speed of the engine rotor.

If $n$ transitions from one motion stage to another take place within the run-up procedure, and if the time value of transition from one stage to another is marked as $t_{1}, t_{2}, \ldots, t_{n}$, then in the course of transition to non-sliding stage

$$
\begin{aligned}
& \varphi_{2}=\varphi_{2}\left(t_{i}\right) ; \varphi_{3}=\varphi_{3}\left(t_{i}\right) ; \varphi_{4}=\varphi_{4}\left(t_{i}\right), \\
& (i=1,3,5, \ldots n) .
\end{aligned}
$$

In case of repeated transition to the sliding stage

$$
\begin{gathered}
\varphi_{1}=\varphi_{1}\left(t_{1}\right)+\sum_{j=2,4, \ldots, i} \varphi_{2}\left(t_{j}\right)-\varphi_{2}\left(t_{j-1}\right) ; \\
\varphi_{2}=\varphi_{2}\left(t_{i}\right) ; \varphi_{3}=\varphi_{3}\left(t_{i}\right) ; \varphi_{4}=\varphi_{4}\left(t_{i}\right) ; \\
\omega_{1}=\omega_{2}\left(t_{i}\right) ; \omega_{2}=\omega_{2}\left(t_{i}\right) ; \omega_{3}=\omega_{3}\left(t_{i}\right) ; \omega_{4}=\omega_{4}\left(t_{i}\right) \\
(i=2,4,6, \ldots, n-1) .
\end{gathered}
$$

\section{CALCULATION EXAMPLE}

Let us consider the UNB-600 drilling pump, equipped with AKZ-15-41-8B asynchronous motor. As long as the combined moment of inertia of the moving parts of the pump is quite significant, an operational air clutch is used for starting the pump. Before the start, the clutch is unlocked and then an idle engine start is carried out. The further system running is carried out by means of gradual engaging of the clutch. A moment of effective resistance force is defined according to the formula (14). The motor parameters are as follows: voltage amplitude of the power circuit $U_{m}=4.9 \mathrm{~kW}$; active resistances of stator and rotor phases $r_{S}=0.38 \Omega, r_{R}=0.318 \Omega$; dissipated inductance $L_{S}=1.048 \cdot 10^{-2} \mathrm{~Hz}, L_{R}=1.112 \cdot 10^{-2} \mathrm{~Hz}$; operational inductance $L_{m}=0.505 \mathrm{~Hz}$; number of magnetic poles pairs $p_{0}=4$; rotor moment of inertia $J_{1}=55 \mathrm{~kg} \cdot \mathrm{sq} . \mathrm{m}$ The drilling pump UNB-600 is a double-cylinder and double-acting pump, consisting of drive and hydraulic parts, which are mounted on a common frame. The angle between the drive links of the slider-crank mechanisms of the pump equals $90^{\circ} \quad\left(\varphi_{1}=\varphi, \varphi_{2}=\varphi+\pi / 2\right)$. Weight of links amounts to: $m_{1}=3850 \mathrm{~kg}, m_{2}=1150 \mathrm{~kg}, m_{3}=420 \mathrm{~kg}$; geometrical dimensions of links: $l_{1}=0.2 \mathrm{~m}$, $a_{1}=0.13 \mathrm{~m}, l_{2}=0.85 \mathrm{~m}, a_{2}=0.25 \mathrm{~m}$; central moments of inertia of the crank and connecting-rod, equal $J_{S 1}=42 \mathrm{~kg}$ sq.m, $J_{S 2}=137 \mathrm{~kg} \cdot \mathrm{sq} \cdot \mathrm{m}$, respectively. Initial conditions of integration of the differential equations (4), (6), (19) for the period of acceleration of the pump by engaging of the operational clutch are following:

$$
\begin{aligned}
& \omega_{1}(0)=78.5 ; \omega_{2}(0)=0 ; \omega_{3}(0)=0 ; \omega_{4}(0)=0 ; \\
& \varphi_{1}(0)=0 ; \varphi_{2}(0)=0 ; \varphi_{3}(0)=0 ; \varphi_{4}(0)=0 ; \\
& i_{S x}(t)=0.071 ; i_{S y}(t)=-30.27 ; i_{R x}(t)=6.777 \cdot 10^{-6} ; \\
& i_{R y}(t)=5.441 \cdot 10^{-6} .
\end{aligned}
$$

The values of currents and angular velocity of the rotor had been received in the course of calculation of the idle motor start before the stable mode was achieved.

As the result of the joint numerical integration of the differential equations of the mechanical system movement (4), (6) or (4), (8) and equations, describing the electromagnetic phenomena in asynchronous motor (19), we receive time dependencies of the following values $\omega_{1}, \omega_{2}, \omega_{3}, \omega_{4}$, electromagnetic moment $M_{E}$, and well as the torques $M_{1 c}$ and $M_{2 c}$ in the elastic links of the unit, which are obtained by the following dependencies:

$$
\begin{aligned}
& M_{1 c}=c_{1}\left(\varphi_{2}-\varphi_{3}\right)+v_{1}\left(\omega_{2}-\omega_{3}\right) ; \\
& M_{2 c}=c_{2}\left(\varphi_{3}-\varphi_{4}\right)+v_{2}\left(\omega_{3}-\omega_{4}\right) .
\end{aligned}
$$

As a result of calculations, the graphic dependencies of an electromagnetic moment, a moment in elastic links of the drive system and acceleration time of a pumping unit on the maximum friction moment in the air clutch were obtained. The calculations were performed for different values of time of filling the clutch with air. The dependencies in Fig. 2 are presented as curves obtained by logarithmic approximation of the results of solving an appropriate system of differential equations of a pumping unit movement.

The calculations of run-up processes were carried out for the pressure on the pumping pistons $5 \mathrm{MPa}$ and time of filling the clutch with air $2,3,4,5$ and 6 seconds. The value of the friction moment of the air clutch was changed in the range from 1000 (or 500) to $10500 \mathrm{Nm}$.

Besides, for the cases of loading the pump with the pressure $5 \mathrm{MPa}$ and time of filling the clutch with air $4 \mathrm{~s}, 5 \mathrm{~s}$ and $6 \mathrm{~s}$, we have received graphic dependencies (Fig. 3) of time of a pumping unit's entering into a stable mode on the friction moment in the clutch changed in the range from 1000 to $10000 \mathrm{Nm}$.

Analyzing the received curves, we see that an increase of the friction moment causes increase of the electromagnetic moment and the moment 
a)

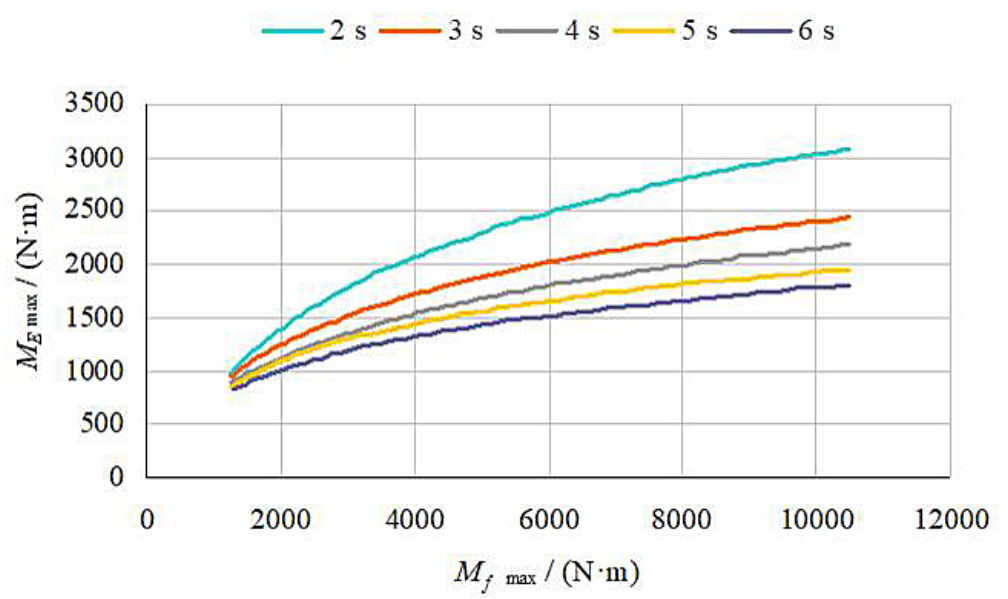

b)

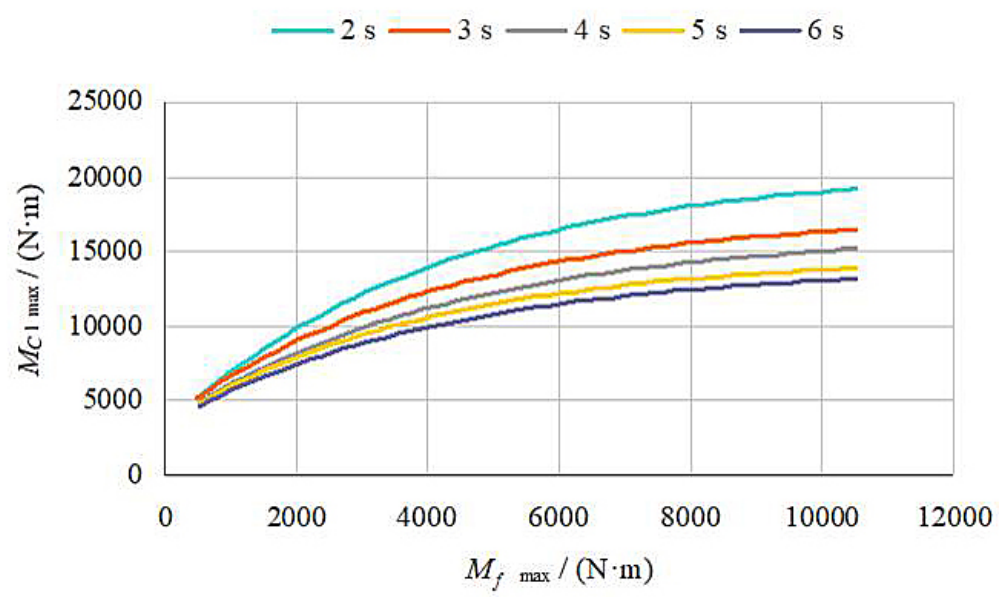

c)

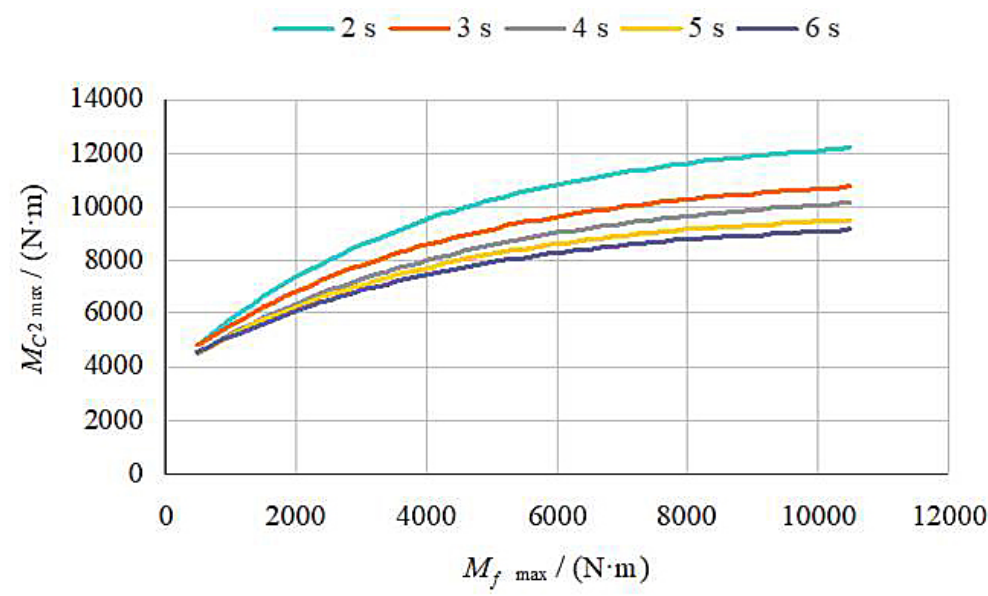

Fig. 2. Graphic dependencies of the maximum value of the electromagnetic moment (a), the moment in the transmission pumping shaft (b) and the moment in the root pumping shaft (c) on the friction moment in the clutch at the pressure on a pumping piston $5 \mathrm{MPa}$ for different time of filling the clutch

in transmission and root shafts of a pumping unit. An important factor that influences the increase of moments in the mechanism links is time of filling the clutch with air. Distinctively, for small friction moments in the clutch at different time of filling the clutch with air, the electromagnetic moment and moments on the driven pulley of belt drive and a gear of a root shaft of cogged gear are practically the same. Approximated curves show that when clutch filling lasts $6 \mathrm{~s}$, the bearing construction elements of the drive mechanism are being less loaded than in case of clutch filling during 2 or $3 \mathrm{~s}$. Thus, the carried-out analysis gives grounds to make a conclusion that the time of filling the clutch with air considerably influences the run-up process of a pumping unit. 


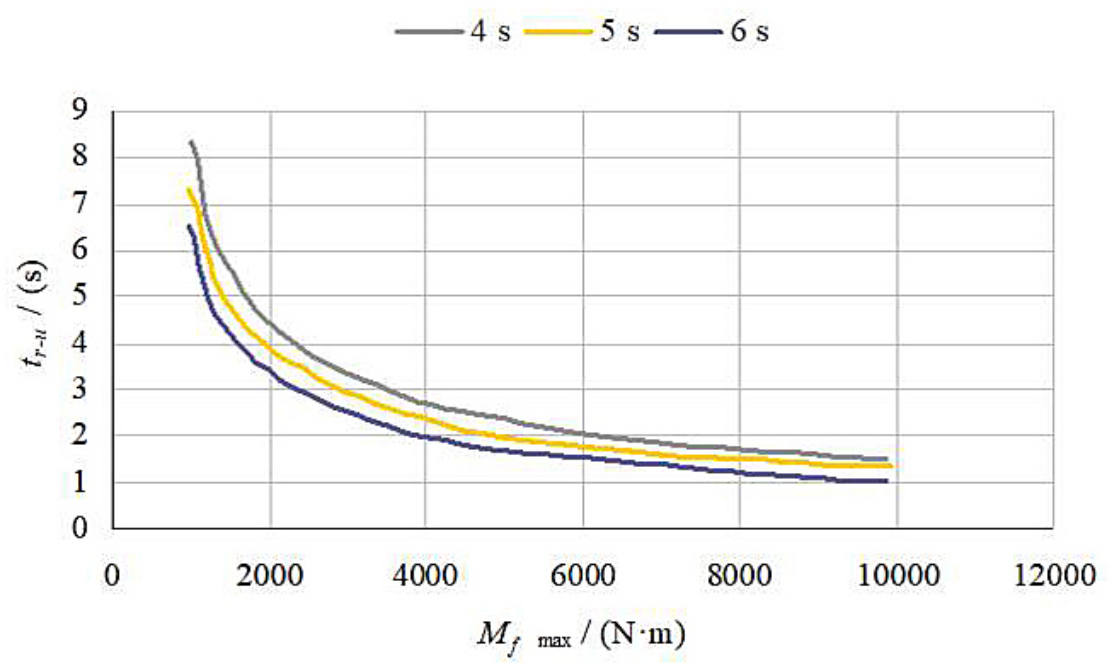

Fig. 3. Graphic dependencies of run-up time of a pumping unit $\left(t_{r-u}\right)$ on the friction moment $\left(M_{f}\right)$ in the air clutch at the pressure on pumping pistons $5 \mathrm{MPa}(a)$ for different time of filling the clutch

\section{CONCLUSIONS}

The run-up time of a pumping unit depends directly on the operation of the friction link of a pumping unit. The sliding duration of operating surfaces of the air clutch is defined by the law of a friction moment change in a clutch, which in its turn depends on the time of filling the clutch with air. As evident from the obtained curves, the runup time of a pumping unit decreases considerably with an increase of the friction moment in the clutch and acceleration of its filling with air. With short duration of filling the clutch with air, there is a possibility of a sharp increase of loading on the elements of the drive mechanism of the pump.

Using the operational friction clutch in the drilling machine pumping unit enables to significantly reduce dynamic loads of the drive system elements. It was established that due to a little increasing of coupling filling time (from 3 to $6 \mathrm{~s}$ ) allows to reduce the stresses in the drive mechanism elements by $20-30 \%$. During the analysis of mathematical modeling results, the rational operational parameters of the shaft-air coupling and the moment of friction and the coupling filling time by air were determined. This will provide a sufficiently rapid acceleration of the unit when the dynamic loads on the pump actuator are limited.

\section{REFERENCES}

1. Badlani M. and Kleinhenz W. Dynamic Stability of Elastic Mechanisms. Journal of Mechanical Design, 101(1), 1979, 149-153.
2. Christoforou A. and Yigit A. Dynamic modeling of rotating drill string with borehole interactions. Journal of Sound and Vibration, 206(2), 1997, 243-260.

3. Dresig H. and Holzweig F. Dynamics of Machinery. Springer, 2010.

4. Erkaya S., Su S., Uzmay I. Dynamic Analysis of a Slider-Crank Mechanism with Eccentric Connector and Planetary Gears. Mechanism and Machine Theory, 42(4), 2007, 393-408.

5. Fung R. F., Chen K. Y., Hsien S. C. Dynamic modelling and identification of a slider-crank mechanism. Journal of Sound and Vibration, 289(4-5), 2006, 1019-1044.

6. Kharchenko E.V. Dynamical processes of drilling rigs. Svit, 1991.

7. Koser K. A slider-crank mechanism based robot arm performance and dynamic analysis. Mechanism and Machine Theory, 39(2), 2004, 169-182.

8. Mudrik I. Measurement of dynamic properties of machine aggregate with variable parameters and asynchronous motor. Journal of Theoretical and Applied Mechanics, 23(1), 1992, 40-41.

9. Patel R. S., Patel D. S., Patel B. D. A Review on Kinematic and Dynamic Analysis of Mechanism. International Journal of Engineering Science and Innovative Technology, 2(2), 2013, 338-341.

10. Viscomi B. V. and Arye R. S. Nonlinear dynamic response of elastic slider crank mechanism. Journal of Engineering for Industry, 93(1), 1971, 251-262.

11. Yutaev V. G. Dynamics of drilling rigs. Nedra, 1987.

12. Zhu H. T., Zhang X., Zhang J. T., Hu S. H. Dynamic simulation of a smart crank and slider mechanism. Journal of Marine Science and Application, 1(2), 2002, 62-65. 